\section{Stem/progenitor cells in the developing human cerebellum: an immunohistochemical study}

\author{
V. Pibiri, ${ }^{1}$ A. Ravarino, ${ }^{1}$ C. Gerosa, ${ }^{1}$ \\ M.C. Pintus, ${ }^{2}$ V. Fanos, ${ }^{2}$ G. Faa ${ }^{1}$ \\ 'Department of Surgical Sciences, \\ Division of Pathology, University of \\ Cagliari \\ 2Department of Surgical Sciences, \\ Neonatal Intensive Care Unit, \\ Puericulture Institute and Neonatal \\ Section, University of Cagliari, Italy
}

\section{Abstract}

The aim of this study was to analyze, by immunohistochemistry, the occurrence of stem/progenitor cells localized in the different niches of the developing human cerebellum. To this end, cerebellar samples were obtained from 3 fetuses and 3 newborns ranging, respectively, from 11 to 24 and from 30 to 38 weeks of gestation. Specimens were $10 \%$ formalin-fixed, routinely processed and paraffinembedded; $3 \mu \mathrm{m}$-tick sections were immunostained with anti-SOX2 and PAX6 antibodies. Our study evidenced SOX2 and PAX6 immunoreactivity in precursors cells in all six developing human cerebella. SOX2 was expressed in precursors of different neural cell types, including Purkinje neurons, stellate cells, basket cells and Golgi cells. In the cerebellar cortex, SOX2 expression changed during gestation, being highly expressed from the $20^{\text {th }}$ up to the $24^{\text {th }}$ week, whereas at the $30^{\text {th }}$ and at the $34^{\text {th }}$ week SOX2 immunoreactivity was restricted to the Purkinje cell layer and the inner zone. Cerebellar human cortex was negative at the $38^{\text {th }}$ week of gestation. PAX6 immunoreactivity was restricted to granule cell precursors in the external granule layer (EGL), being detected at all gestational ages. Our study indicates SOX2 and PAX6 as two useful markers of stem/progenitor cells that highlight the different germinative zones in the developing human cerebellum.

\section{Introduction}

During development, all cerebellar cells originate from two distinct germinative zones: the neuroepithelium surrounding the IV ventricle and theexternal granule layer (EGL). ${ }^{1}$ Niches of cerebellar neural stem cells are localized in these germinative areas. Neural stem cells (NSCs) are the main source of all different cell types of the central nervous system (neurons and glia), both in the developing and in the adult brain. They are characterized by the capacity of self-renewal and the ability to differentiate towards the multiple cell types that characterize the mature cerebellum.

\section{The proliferative neuroepithelium} during cerebellar development

Formed by pseudo-stratified neuroepithelium, the ventricular zone (VZ) of this neuroepithelium is composed of neuroepithelial cells that are considered the primary progenitors of several cerebellar cell types. Cerebellar progenitors from the neuroepithelium differentiate into cerebellar GABAergic neurons, including neurons of the deep nuclei, Purkinje cells and inhibitory interneurons (basket cells, stellate cells, candelabrum cells and Golgi cells). ${ }^{2}$ Experimental studies have demonstrated that, in the developing brain, migration of neuronal precursors is driven by a particular type of glial cells, the radial glia, that originate from the ventricular neuroepithelium at the early stages of development and act as a scaffold along which precursors climb during their radial migration towards the pial surface.,4

\section{The external granule layer as a} niche in the developing cerebellum

At the early stages of cerebellar neurogenesis, progenitor cells migrate out of the rhombic lip and spread across the cerebellar surface to form a secondary neurogenic zone, the EGL, ${ }^{5}$ a unique peculiarity within the developing CNS. The EGL is a transitional zone of proliferating cells, localized below the pial surface and composed of dividing granule cells precursors (GCPs) that give rise to the granule cells of the cerebellum, the most abundant neurons in the mammalian brain. ${ }^{6}$ In the EGL, post-mitotic GPCs begin to extend their axons along Bergmann fibers, a unipolar specialized protoplasmatic astrocytes located in the cerebellar cortex. ${ }^{7}$ At the level of Purkinje cell layer, GPCs detach from the radial Bergmann fibers and move inward to form the inner internal granule layer (IGL), differentiating into mature granule cells. ${ }^{8} \mathrm{GPC}$ proliferation and migration actively occurs during all gestation, going on in early post-natal life.

\section{SOX2 expression in stem/progenitor cells in the neuroepithelium}

The transcription factor SOX2 [Sex determining region of Y chromosome (Sry)-related high mobility group box2] belongs to the SOX family of transcription factors characterized by the presence of a homologous sequence known as HMG (high mobility group) box, a DNA binding domain highly conserved among species. ${ }^{9-11}$ SOX genes encode putative transcriptional regulators implicated in cell fate
Correspondence: Valeria Pibiri, Department of Surgical Sciences, Division of Pathology, San Hospital Giovanni di Dio, University of Cagliari, via 0spedale 54, 09124 Cagliari, Italy. Tel. +39.070 .6092370 - Fax: +39.070 .6092115 .

E-mail: valeria.pibiri@libero.it

Key words: Human cerebellum; human fetus; immunohistochemistry; stem cells; human development; cerebellar niches.

Contributions: VP, GF, research design; VP, AR, GF, experiments performing; VP, AR, CG, MCP, contribution to experiment results interpretation; VP, GF, manuscript writing; VF, GF, research coordination, contribution to final revision of the paper.

Conflict of interest: the authors declare no conflict of interest.

Acknowledgments: the authors are grateful to Prof. Giacomo Cao (Dept. of Chemical and Materials Engineering, University of Cagliari) for relevant critical suggestions. V. Pibiri has performed her activity in the framework of the International PhD in Innovation Sciences and Technology at the University of Cagliari, Italy. V. Pibiri gratefully acknowledges Sardinia Regional Government for the financial support of her PhD scholarship (P.O.R. Sardegna F.S.E. Operational Programme of the Autonomous Region of Sardinia, European Social Fund 2007-2013 - Axis IV Human Resources, Objective 1.3, Line of Activity l.3.1.).

Received for publication: 23 May 2016.

Accepted for publication: 30 July 2016.

This work is licensed under a Creative Commons Attribution-NonCommercial 4.0 International License (CC BY-NC 4.0).

(C) Copyright V. Pibiri et al., 2016

Licensee PAGEPress, Italy

European Journal of Histochemistry 2016; 60:2686 doi:10.4081/ejh.2016.2686

during development and in different developmental processes control. ${ }^{12,13}$ Experimental studies showed that SOX2 is expressed during the development of the mammalian central nervous system. ${ }^{14-16}$ In mice, the expression of SOX2 persists also in adulthood: SOX2 expression has been reported in neural precursors located in the subventricular zones as well as in the subgranular zone of the hippocampus. ${ }^{17}$ Furthermore, SOX2-positive cells have been reported in different regions of the adult mouse brain, including the cerebral cortex, the subventricular zone of the lateral ventricles, thalamus and striatum. ${ }^{18}$ In humans, SOX2 expression has been reported in the Purkinje cell layer of the adult cerebellum, suggesting that the maintainance of this marker might be typical of the adult cerebellum. ${ }^{19}$ Recent data 
from our group showed the presence of SOX2 expression in the subventricular zone of the lateral ventricle in the adult (L. Vinci, unpublished data).

The function of SOX2 has been investigated using several transgenic mice models. Experimental studies demonstrated that a constitutive expression of SOX2 maintains the properties of stem cells and inhibits neuronal development. On the other hand, in these transgenic mice, the inactivation of SOX2encoding genes resulted in a early exit of neural precursors from the cell cycle. This was associated with a loss of progenitor markers and the onset of early neuronal differentiation markers..$^{20}$ Additionally, SOX2 expression was lost during neuronal differentiation. These data suggest that SOX2 is involved in the maintenance of the stemness identity of the NSCs. At the best of our knowledge, in humans few data have been reported regarding the expression of SOX2 in the developing human nervous system and, in particular, in the human cerebellum. ${ }^{21}$

\section{PAX6, a marker for granule cells precursors in the EGL}

PAX6 (paired box gene 6) belongs to the family of PAX gene class and encodes for a transcription factor containing a paired domain and a paired-type homeodomain. ${ }^{22}$ PAX6 plays a critical role in brain and eye development. ${ }^{23-26}$ In the CNS, it is involved in neuronal specification, neuronal migration and axonal extension. ${ }^{27,28}$ In the mouse cerebellum, PAX6-immunoreactive cells were found in migrating granule cell precursors from the EGL. ${ }^{29}$ PAX6 immunoreactivity coincides with the development of the EGL on the cerebellar surface and it is detected from the early stages of gestation till the first post-natal months.

On the basis of experimental studies, the purpose of this study was to investigate the occurrence of stem/progenitor cells in the human fetal cerebellum, using two molecular markers, SOX2 and PAX6, for the identification of the different neural cerebellar niches during development.

\section{Materials and Methods}

Cerebellum samples were obtained from 6 human fetuses and newborns ranging from 11 to 38 weeks of gestation, received from the Obstetric Division and from the Neonatology Department of the University of Cagliari. All the fetuses included in this study had no congenital brain malformation. Regarding the cause of death, the 11 week-old fetus underwent voluntary termination of pregnancy
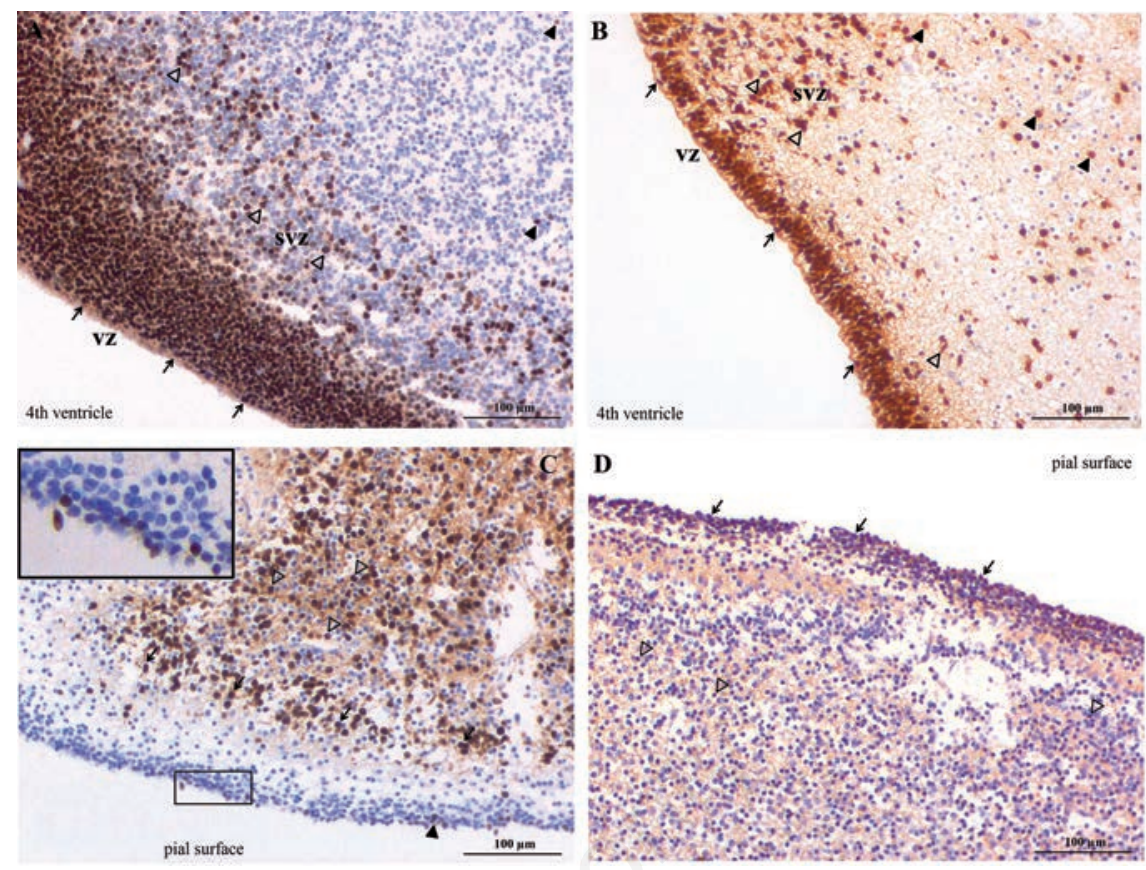

Figure 1. A) Immunoreactivity for SOX2 in progenitor cells in the ventricular zone (VZ, arrows), in the subventricular zone (SVZ; open arrowheads) and scattered positive cells in the inner zone (arrowheads) in a 11-week-old fetus and B) in a 20-week-old fetus. C) SOX2 immunoreactivity in the Purkinje cell layer (arrows), in the internal zone (open

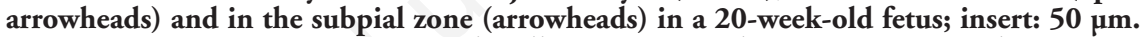
D) PAX6 immunoreactivity in granule cell precursors in the EGL (arrows) and in migrating granule cell precursors in the internal zone (open arrowheads) in a 20-week-old fetus.
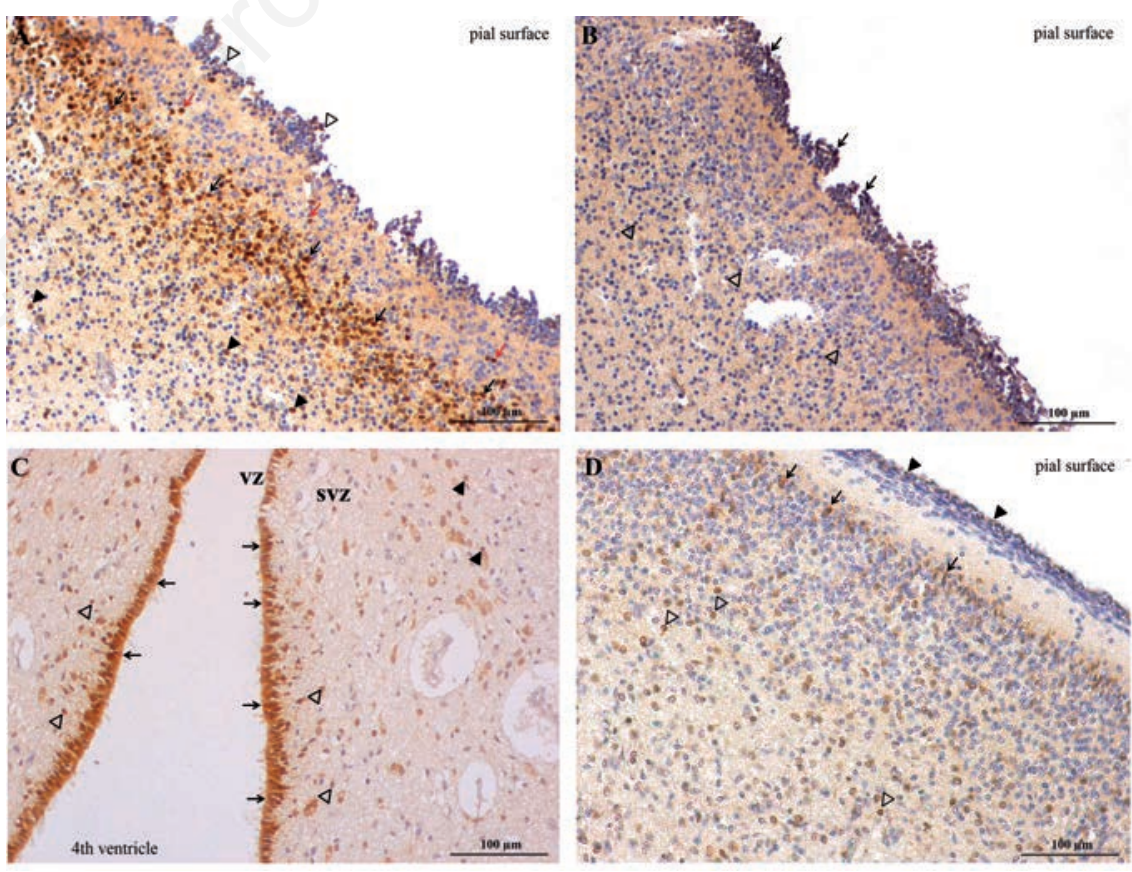

Figure 2. A) SOX2 immunoreactivity in the Purkinje cell layer (arrows), in the subpial zone (open arrowheads), in the internal zone (arrowheads) and scattered positive cells in the molecular layer (red arrows) at 24 weeks of gestation. B) PAX6 immunoreactivity in granule cell precursors in the EGL (arrows) and in migrating granule cell precursors in the internal zone (open arrowheads) at 24 weeks of gestation. C) Immunoreactivity for SOX2 in progenitor cells in the VZ (arrows), in the subventricular zone (SVZ; open arrowheads) and scattered immune-positive cells in the inner zone (arrowheads) in a 30week-old fetus. D) SOX2-positive cells in the Purkinje cell layer (arrows), in the inner zone (open arrowheads) and in the subpial zone (arrowheads) in a 30-week-old fetus. 
(VTOP); the 20 week-old fetus underwent therapeutic abortion following the diagnosis of diaphragmatic hernia; placental detachment was the cause of death in the 24 week-old fetus, in fetuses of 30,34 and 38 weeks of gestation the cause of death was sepsis. All procedures were approved by the Ethics Human Studies Committee of University Medical Centre of Cagliari (according to the instructions of the Declaration of Helsinki). Samples were fixed in $10 \%$ buffered formalin, routinely processed, and paraffin-embedded. Serial 3 $\mu \mathrm{m}$-thick sections were obtained from each paraffin block; after dewaxing and rehydrating, one of these was stained with hematoxylineosin, while the others were pre-treated for immunohistochemical analysis, with $10 \mathrm{~min}$ heat-induced epitope retrieval in buffer $\mathrm{pH}$ 9.00 (EnVision $^{\mathrm{TM}}$ FLEX Target Retrieval Solution High pH; Dako Denmark A/S, Glostrup, Denmark; code K8004). Slides were then incubated for $20 \mathrm{~min}$ at room temperature with anti-SOX2 (SRY-box 2; Santa Cruz Biotechnology, Dallas, TX, USA; Code SC365823; mouse monoclonal antibody clone E-4 at 1:50 dilution) and anti-PAX6 (Santa Cruz Biotechnology; code SC-53108; mouse monoclonal antibody at 1:50 dilution). As a negative control, cerebellar sections were incubated in dilution buffer without primary antibody. Staining procedures were performed by Envision $^{\text {TM }}$ FLEX+ (Dako; code K8002) Detection System and AutostainerLink 48 instrument following dealer's instructions. Data were obtained by evaluation of positivity (+) and negativity (-) for SOX2 and PAX6 immunoreactivity in each cerebellar sample.

\section{Results}

Immunoreactivity for SOX2 and PAX6 was detected in all of six developing human cerebellum samples analyzed in this study. Differences were found regarding SOX2 and PAX6 immunostaining in the different germinative areas of these organs.

- At 11 weeks of gestation, SOX2 was expressed in the nuclei of stem/progenitor cells of the neuroepithelium surrounding the fourth ventricle (Figure 1A). A strong immunoreactivity for SOX2 was observed at nuclear level in the vast majority of cerebellar progenitors of the ventricular and subventricular zone. Scattered immunoreactive cells were also detected in the inner zone, suggesting the migration of neuronal precursors towards the pial zone. No reactivity for PAX6 was observed at this gestational age.

- In the cerebellar cortex at 20 weeks of gestation, SOX2 immunoreactivity was found in the nuclei of stem/progenitor cells of the cere- bellar neuroepithelium in the ventricular zone, in migrating progenitor cells in the subventricular zone and scattered immune-positive cells were also found in the inner zone (Figure 1B). S0X2 expression was mainly detected in nuclei of neural precursors localized in the Purkinje cell layer (Figure 1C). SOX2-positive cells were also found in the internal granule layer of the developing cerebellum. A mild reactivity for SOX2 was observed in a minor part of cerebellar precursors in the subpial zone. At this gestational age, an evident nuclear immunoreactivity for PAX6 was found in granule cell precursors of the EGL (Figure 1D). Scattered PAX6-positive cells were also detected at the inner zone, suggesting the migration of these cells towards the internal granule layer.

- In the cerebellar cortex at 24 weeks of gestation, SOX2 immunostaining was mainly found in the nuclei of neural progenitors in the Purkinje cell layer and in the inner zone (Figure 2A). A mild reactivity for SOX2 was observed in progenitor cells in the subpial zone. Scattered S0X2-positive cells were also observed in the molecular layer. At this gestational age, nuclear PAX6 immunostaining highlighted granule cell precursors in the cerebellar EGL (Figure 2B). Scattered PAX6-positive cells were also detected in the inner zone, suggesting the migration of these progenitor cells towards the internal granule layer.

- In the cerebellar cortex at 30 weeks of gestation, immunoreactivity for SOX2 was found in the nuclei of stem/progenitor cells in the neuroepithelium of the ventricular zone and in migrating progenitors in the subventricular zone. Immunoreactivity for SOX2 was also found in precursor cells migrating towards the pial zone Figure 2C). SOX2 expression was also detected in a percentage of cerebellar neural precursors in the Purkinje cell layer and in the inner zone (Figure 2D). A mild reactivity for SOX2 was observed in progenitor cells in the subpial zone. PAX6-positive cells were found in the EGL precursors layer (Figure 3A). Scattered PAX6-positive cells were also detected in the inner zone, suggesting the migration

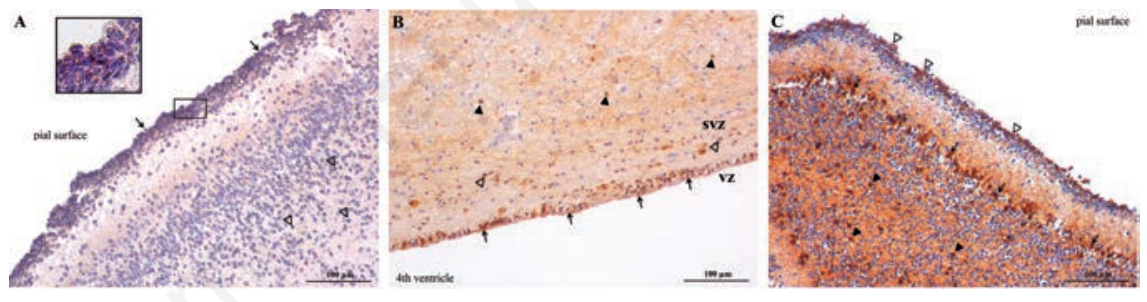

Figure 3. A) PAX6-positive cells in granule cell precursors in the EGL (arrows) and in the internal zone (open arrowheads) in a 30-week-old fetus; insert: $50 \mu \mathrm{m}$. B) Immunoreactivity for SOX2 in progenitor cells in the VZ (arrows), in the subventricular zone (SVZ; open arrowheads) and scattered immune-positive cells in the inner zone (arrowheads) in a 34-week-old fetus. C) SOX2 immunoreactivity in the Purkinje cell layer (arrows), in the subpial zone (open arrowheads) and scattered positive cells in the inner zone (arrowheads) in a 34-week-old fetus.

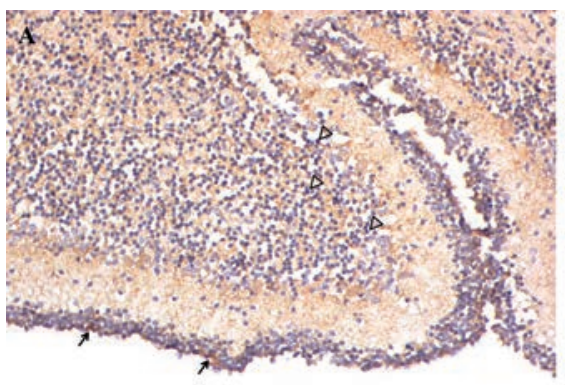

pial surface

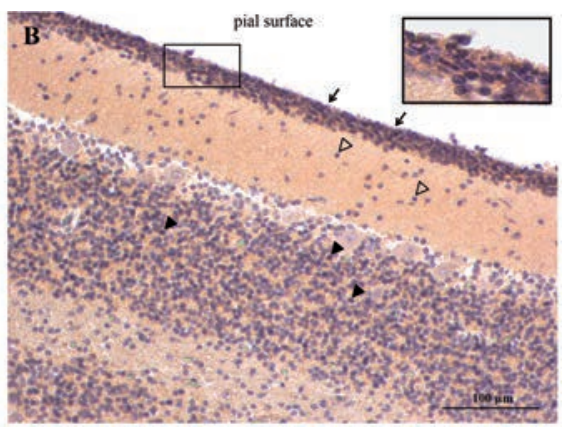

Figure 4. A) PAX6 immunoreactivity in granule cell precursors in the EGL (arrows) and in migrating granule cell precursors in the internal granule layer (open arrowheads) in a 34 week-old fetus. B) PAX6 immunoreactivity in granule cell precursors in the EGL (arrows), scattered positive cell in the molecular layer (open arrowheads) and migrating granule cell precursors in the internal granular layer (arrowheads) at the $38^{\text {th }}$ gestation week; insert: $50 \mu \mathrm{m}$. 
of these cells towards the internal granule layer.

- In the cerebellar cortex at 34 weeks of gestation, immunoreactivity for SOX2 was detected at nuclear level of stem/progenitor cells in the neuroepithelium of the ventricular zone, in migrating progenitors in the subventricular zone and in the inner zone (Figure 3B). A strong reactivity for SOX2 was detected in a percentage of cerebellar precursors in the Purkinje cell layer (Figure 3C). Moreover, a mild reactivity for SOX2 was observed in neural precursors in the subpial zone. Scattered SOX2-positive cells were also found in the inner zone. At this gestational age, a persistent immunoreactivity for PAX6 was mainly observed in the precursors of the EGL (Figure 4A). Scattered migrating PAX6-immunoreactive cells were found in the molecular layer.

- In the cerebellar cortex at 38 weeks of gestation, SOX2-positive cells were not found in any of the cortical layers of the developing cerebellum. Conversely, PAX6 nuclear immunostaining was observed in granule cell precursors in the EGL (Figure 4B). Immunoreactivity for PAX6 was also detected in the molecular layer. Purkinje cell layer was negative.

\section{Discussion}

Previous studies on stem/progenitor cells in the central nervous system have been mainly focused on animal models; these studies have shown that SOX2 is involved in the development of the mammalian central nervous system. During neurogenesis, this marker has been reported to be expressed in the developing neural tube as well as in proliferating progenitor cells..$^{30}$ In our study we demonstrated that, during human cerebellar development, SOX2 is intensively expressed both in the nuclei of progenitor cells of the neuroepithelium of the fourth ventricle and in the nuclei of progenitors of the cerebellar cortex migrating from the neuroepithelium towards the cortical surface. Immunoreactivity for SOX2 was observed from the $20^{\text {th }}$ up to $24^{\text {th }}$ week of gestation. At the $30^{\text {th }}$ and $34^{\text {th }}$ week SOX2 immunoreactivity was restricted to the Purkinje cell layer and the inner zone, whereas at the $38^{\text {th }}$ week of gestation we did not detect SOX2 cortical expression. On the basis of these findings, we can speculate that SOX2 in human developing cerebellum is down-regulated when progenitors exit from cell cycle and become post-mitotic. These data confirm previous experimental studies on transgenic mice models, in which the inactivation of the SOX2encoding gene paralleled the exit of neural precursors from the cell cycle. SOX2-silencing was associated with a loss of progenitor markers and the onset of the early expression of neuronal differentiation markers. ${ }^{31}$ Further studies on SOX2 expression in the earliest stages of human development are needed in order to confirm the hypothesis that SOX2 may represent a marker of the cerebellum development also at embryonic stage (first 8 weeks of gestation).

Another interesting finding emerging from our study is the immunoreactivity for PAX6 of granular precursor cells in the developing cerebellum. Nuclear expression of PAX6 was observed from 20 to 38 weeks of gestation, reactivity being restricted to the external granular layer. As previously mentioned, PAX6 is crucial for eye development, ${ }^{32}$ and a mutation in PAX6 gene was responsible for aniridia, ${ }^{33}$ in pancreatic islets ${ }^{34}$ and central nervous system development. ${ }^{35}$ Experimental studies demonstrated that, in the developing mouse cerebellum, PAX6 is expressed in granule cell progenitors in the external granular layer and in granule cell progenitors that are migrating from the external toward the internal granular layer. ${ }^{36}$ Our study demonstrates that, in the development of human cerebellum, precursor cells of the external granular layer are PAX6+ and give rise to the granular cells of the human cerebellum. We also observed immunoreactivity for PAX6 both in granule cell precursors in the EGL and in migrating granule cell precursors in the IGL, from the $20^{\text {th }}$ gestational week to term. After birth, the external granular layer decreases in thickness and disappears during the $1^{\text {st }}$ postnatal year. ${ }^{37}$ We have found PAX6 immunoreactivity in human cerebellar cortex of 4 and 6 postnatal months (data not shown), but the thickness of the EGL was strongly reduced. These data confirm previous experimental studies regarding PAX6 immunoreactivity in the developing cerebellum, suggesting that PAX6 may represents a key regulator during the development of the human cerebellum. In conclusion, our study indicates SOX2 and PAX6 as two useful markers for the identification of stem/progenitor cells in the developing human cerebellum. Further studies are needed in order to identify other markers of stem/progenitor cells during embryo-fetal development of the human cerebellum.

\section{References}

1. Wingate RJ. The rhombic lip and early cerebellar development. Curr Opin Neurobiol 2001;11:82-8

2. Hoshino M, Nakamura S, Mori K, Kawauchi T, Terao M, Nishimura YV, et al. Ptfla, a bHLH transcriptional gene, defines GABAergic neuronal fates in cere- bellum. Neuron 2005; 47:201-13.

3. Rakic P. Mode of cell migration to the superficial layers of fetal monkey neocortex. J Comp Neurol 1972;145:61-83.

4. Malatesta P, Appolloni I, Calzolari F. Radial glia and neural stem cells. Cell Tissue Res 2008;331:165-78.

5. ten Donkelaar HJ, Lammens M, Wesseling P, Thijssen HO, Renier WO. Development and developmental disorders of the human cerebellum. J Neurol 2003;250:1025-36.

6. Alder J, Cho NK, Hatten ME. Embryonic precursors cells from the rhombic lip are specified to a cerebellar granule neuron identity. Neuron 1996;17:389-99.

7. Buffo A, Rossi F. Origin, lineage and function of cerebellar glia. Prog Neurobiol 2013;109:42-63.

8. Komuro H, Yacubova E, Yacubova E, Rakic P. Mode and tempo of tangential cell migration in the cerebellar external granular layer. J Neurosci 2001;21:527-40.

9. Laudet V, Stehelin D, Clevers H. Ancestry and diversity of the HMG box superfamily. Nucleic Acids Res 1993;21:2493-501.

10. Koopman P. Sry and Sox9: mammalian testis-determining genes. Cell Mol Life Sci 1999;55:839-856.

11. Weiss MA. Floppy SOX: mutual induced fit in hmg (high-mobility group) box-DNA recognition. Mol Endocrinol 2001;15:353-62.

12. Arsic N, Rajic T, Stanojcic S, Goodfellow PN, Stevanovic M. Characterization and mapping of the human SOX14 gene. Cytogenet Genome Res 1998;83:1-2.

13. Sarkar A and Hochedlinger K. The SOX family of transcription factors: versatile regulators of stem and progenitor cell fate. Cell Stem Cell 2013;12:15-30.

14. Bani-Yaghoub M, Tremblay RG, Lei JX, Zhang D, Zurakowski B, Sandhu K, et al. Role of Sox2 in the development of the mouse neocortex. Dev Biol 2006;295:52-66.

15. Sasai Y. Roles of Sox factors in neural determination: conserved signaling in evolution? Int J Dev Biol 2001;45:321-6.

16. Feng R, Wen J. Overview of the roles of Sox2 in stem cells and development. Biol Chem 2015;396:883-91.

17. Alcock J, Lowe J, England T, Bath P, Sottile V. Expression of Sox1, Sx2 and Sox9 is maintened in adult human cerebellar cortex. Neurosci Lett 2009;450:114-6.

18. Thiel G. How Sox2 maintains neural stem cell identity. Biochem J 2013;450:1-2.

19. Ferri AL, Cavallaro M, Braida D, Di Cristofano A, Canta A, Vezzani A, et al. Sox2 deficiency causes neurodegeneration and impaired neurogenesis in the adult mouse brain. Development 2004;131:3805-19.

20. Graham V, Khudyakov J, Ellis P, Pevny L. SOX2 functions to maintain neural pro- 
genitor identity. Neuron 2003;39:749-65.

21. Kelberman D, de Castro SC, Huang S, Crolla JA, Palmer R, Gregory JW, Taylor D, et al. SOX2 plays a critical role in the pituitary, forebrain, and eye during human embryonic development. J Clin Endocri Metab 2008;93:1865-73.

22. Callaerts P, Halder G, Gehring WJ. PAX-6 in development and evolution. Annu Rev Neurosci 1997;20:483-532.

23. Manuel MN, Mi D, Mason J0, Price DJ. Regulation of cerebral cortical neurogenesis by the Pax6 transcription factor. Front Cell Neurosci 2015;9:70.

24. Georgala PA, Carr CB. Price DJ. The role of Pax6 in forebrain development. Dev Neurobiol 2011;71:690-709.

25. Grindley JC, Davidson DR, Hill RE. The role of Pax-6 in eye and nasal development. Development 1995;121:1433-42.

26. Shaham 0, Menuchin Y, Farhy C, AsheryPadan R. Pax6: a multi-level regulator of ocular development. Prog Retin Eye Res
2012;31:351-76.

27. Matsumoto Y, Osumi N. [The role of Pax6 in the developing central nervous system].[Article in Japanese]. Brain Nerve 2008;60:365-74.

28. Mastick GS, Andrews GL. Pax6 regulates the identity of embryonic diencephalic neurons. Mol Cell Neurosci 2011;17:190-207.

29. Chung SH, Kim CT, Yung YH, Lee NS, Jeong YG. Early cerebellar granule cell migration in the mouse embryonic development. Anat Cell Biol 2010;43:86-95 .

30. Ellis P. SOX2, a persistent marker for multipotent neural stem cells derived from embryonic stem cells, the embryo or the adult. Dev Neurosci 2004;26:148-65.

31. Graham V, Khudyakov J, Ellis P, Pevny L. SOX2 functions to maintain neural progenitor identity. Neuron 2003; 39:749-765

32. Hanson I, Van Heyningen V. Pax6:more than meets the eye. Trends Genet 1995;11:268-72.

33. Hanson IM, Seawright A, Hardman K,
Hodgson S, Zaletayev D, Fekete G, et al. PAX6 mutations in aniridia. Hum Mol Genet 1993;2:915-20.

34. Beimesche $S$, Neubauer A, Herzig $S$, Grzeskowiak R, Diedrich T, Cierny I, et al. Tissue-specific transcriptional activity of pancreatic islet cell-specific enhancer sequence/Pax6-binding site determined in normal adult tissues in vivo using transgenic mice. Mol Endocrinol 1999;13:718-28.

35. Engelkamp D, Rashbass P, Seawright A, van Heyningen V. Role of Pax6 in development of the cerebellar system. Development 1999;126:3585-96.

36. Yamasaki T, Kawaji K, Ono K, Bito H, Hirano T, Osumi N, et al. Pax6 regulates granule cell polarization during parallel fiber formation in the developing cerebellum. Development 2001;128:133-44.

37. Volpe JJ. Cerebellum of the premature infant: rapidly developing, vulnerable, clinically important. J Child Neurol 2009;24: 1085-104. 